\title{
Efeitos do método Watsu em paciente asmático moderado - relato de caso
}

\author{
Effect of the Watsu method in moderate asthmatic patient - case report
}

\section{Ana Angélica Ribeiro de Lima', Kátia de Souza Pereira ${ }^{2}$, Rosangela Vinhas ${ }^{3}$}

\begin{abstract}
RESUMO
O presente estudo tem como objetivo avaliar os efeitos do Método Watsu na mobilidade torácica, ventilação, estado de ansiedade, depressão e qualidade de vida de paciente asmático moderado. Um indivíduo foi submetido a 10 sessões do Método Watsu, durante 5 semanas.Aavaliaçãoprée pós-tratamentoconsistiunaaplicaçãode questionários para quantificar os sinais e sintomas da asma, estados deansiedadeedepressão, qualidadedevida, avaliaçãoda mobilidade torácica e capacidade pulmonar. Obteve-se ganho nas medidas axilar e xifóide de cirtometria torácica. Nos valores espirométricos, houve aumento do Volume Expiratório Forçado no Primeiro Segundo, relação Volume Expiratório Forçado no Primeiro Segundo / Capacidade Vital Forçada e Pico de Fluxo Expiratório. Ocorreu meIhora naansiedademomentâneae noquestionário dequalidadede vida, com diminuição do escore, nos domínios de limitação das atividadesfísicas ena freqüência egravidade dos sintomas, eaumento naadesãoaotratamento, assim como no domínio sócio-econômico e psico-social. O Método Watsu mostrou-se benéfico, neste estudo de caso, em relação à mobilidade torácica, ventilação, ansiedade e qualidade de vida.
\end{abstract}

Unitermos. Hidroterapia, Asma, Ansiedade, Depressão, Qualidade de Vida.

Citação. Lima AAR, Pereira KS, Vinhas R. Efeitos do método Watsu em paciente asmático moderado - relato de caso.

Trabalho realizado na Universidade Cidade de São Paulo UNICID, São Paulo-SP, Brasil.

1.Fisioterapeuta, Pós-Graduada em Hidroterapia. UNICID (Universidade Cidade de São Paulo), São Paulo-SP, Brasil.

2.Fisioterapeuta, Mestre em Fisiopatologia Experimental, Docente da Universidade Metodista de Piracicaba - UNIMEP, Piracicaba-SP, Brasil.

3.Fisioterapeuta, Pós-Graduada em Hidroterapia, Docente da Universidade Cidade de São Paulo - UNICID, São Paulo-SP, Brasil.

\section{SUMMARY}

The present study has as objective to evaluate the effect of the Watsu Method in the thoracic mobility, ventilation, state of anxiety, depression and quality of life of moderateasthmaticpatients. An individual was submitted to 10 sessions of the Watsu Method during 5 weeks. The preand post-treatment evaluation consisted ofquestionnairestoquantifythesignalsandsymptoms of asthma, states of anxiety, depression, quality of life, evaluation of thoracic mobility and pulmonary capacity. There was an increase in thoracic cirtometry axillary and xiphoid measures. In the spirometric values, there was an increase in Forced Expiratory Volume in the First Second, relation Forced Expiratory Volume in the First Second / Forced Vital Capacity and Peak Expiratory Flow. There was an improvement in the temporary anxiety occurred and in the quality of life questionnaire, there was reduction in the score, as regards limitation of the physical activities and in the frequency and severity of the symptoms. Also, there was increase in adherence to the treatment, as well as in the social-economic and psycho-social domain. The Watsu Method revealed beneficial, as regards thoracic mobility, ventilation, anxiety and quality of life.

Keywords. Hydrotherapy, Asthma, Anxiety, Depression, Quality of Life.

Citation. Lima AAR, Pereira KS, Vinhas R. Effect of the Watsu method in moderate asthmatic patient - case report.

Endereo para correspondência:

Ana Angélica R Lima

R. Onze de Fevereiro, 428

CEP 04319-020, São Paulo-SP, Brasil.

E-mail: ana_rlima@hotmail.com

Recebido em: 28/09/2007

Revisado em: 29/09/2007 a 13/04/008

Aceito em: 14/04/2008

Conflito de interesses: não 


\section{INTRODUÇÃO}

Asma é uma doença inflamatória crônica, caracterizada por hiperresponsividade das vias aéreas inferiores e por limitação variável ao fluxo aéreo'. Acredita-se que $7,2 \%$ da população mundial seja asmática. A prevalência da asma tem aumentado em todo mundo, com o Brasil ocupando o $8^{\circ}$ lugar. Estima-se que existam no Brasil 18 milhões de pessoas com asma, com uma média de 2000 óbitos por ano no país ${ }^{2,3}$.

Com a deterioração gradual imposta pela doença obstrutiva, o paciente apresenta "perdas" em diversas áreas, como lazer, social, profissional, sexual e interpessoal, apresentando com grande prevalência de transtornos ansiosos e depressivos ${ }^{4,5}$.

Watsu ou Water Shiatsu, tem como base os alongamentos e movimentos do Zen Shiatsu, técnica de solo, atuando em todos os níveis do ser humano: emocional, psicológico, espiritual e físico ${ }^{6,7}$.

Este trabalho tem o objetivo de avaliar os efeitos do Método Watsu na mobilidade torácica, ventilação, estado de ansiedade, depressão e qualidade de vida de paciente asmático moderado, através de um estudo de caso.

\section{RELATO DE CASO}

A pesquisa foi realizada no setor de Hidroterapia da Universidade Cidade de São Paulo - UNICID, sendo previamente aprovada pela Comissão de Ética em Pesquisa em Seres Humanos.

Este estudo apresentou um indivíduo do sexo feminino, com 21 anos, com diagnóstico de asma persistente moderada. $\mathrm{O}$ indivíduo foi inicialmente submetido à aplicação do termo de consentimento livre e esclarecido. Através da espirometria, foram avaliadas as medidas de: Capacidade Vital Forçada (CVF), Volume Expiratório Forçado no Primeiro Segundo (VEF1), relação Volume Expiratório Forçado no Primeiro Segundo/Capacidade Vital Forçada (VEF1/CVF) e Pico de Fluxo Expiratório (PFE), sendo realizadas três medidas e selecionada a melhor, com o objetivo de classificar a asma. Foi realizada cirtometria torácica pelo Método Kakizaki ${ }^{8}$. Foram aplicados o Questionário de Qualidade de Vida na Asma (QQV) ${ }^{9}$; o Inventário de Depressão de Beck (IDB) ${ }^{10}$; o Inventário de Ansiedade IDATE-TRAÇO (AIT) de Spielberger ${ }^{11}$.

Em cada sessão, foi realizada a mensuração da freqüência cardíaca, freqüência respiratória, saturação periférica de oxigênio, para controle pré e pós-intervenção. O Inventário de Ansiedade IDATE-ESTADO (AIE) foi utilizado apenas na primeira, sexta e décima sessões afim de avaliar a ansiedade imediata antes e após tratamento.

O programa de tratamento proposto foi de duas sessões semanais de aplicação do Método Watsu em piscina aquecida $\left( \pm 34^{\circ} \mathrm{C}\right)$, com duração de 60 minutos cada, durante 5 semanas completando um total de 10 sessões.

Ao término das 10 sessões de tratamento, foi realizada uma nova Prova de Função Pulmonar e aplicados novamente os questionários de QQV, AIT, IDB e cirtometria torácica.

\section{RESULTADOS}

Nos valores de cirtometria torácica, pré e pós-tratamento, ocorreu aumento na medida axilar, sendo na $1^{\circ}$ expiração de $2 \mathrm{~cm}$, na inspiração de $3 \mathrm{~cm}$ e na $2^{\circ}$ expiração de $5 \mathrm{~cm}$. $\mathrm{Na}$ medida xifóide, ocorreu um aumento na $1^{\circ}$ expiração de $7 \mathrm{~cm}$, na inspiração de $5,5 \mathrm{~cm}$ e na $2^{\circ}$ expiração de $6,5 \mathrm{~cm}$. Na medida umbilical, ocorreu apenas de $0,5 \mathrm{~cm}$ na $2^{\circ}$ expiração (Figura 1 ).

Nos valores espirométricos, pré e pós-tratamento, pode-se observar um aumento de $300 \mathrm{ml} \mathrm{no}$ VEF1 (12\%); aumento de 8,29L no VEF1/CVF (11\%); aumento de $102,1 \mathrm{~L} / \mathrm{min}$ no PFE (39\%). A CVF apresentou uma pequena diminuição $(-0,3 \%)$ (Tabela 1$)$.

No AIE, ocorreu queda de 9 pontos, na primeira sessão. Na sexta sessão, queda de 14 pontos e na décima sessão, uma diminuição de 11 pontos. Nos três resultados, a paciente modificou o escore do estado de ansiedade para de não ansiedade ao final da sessão.

\begin{tabular}{|l|l|l|l|}
\hline & Adequado & Inicial & Final \\
\hline Capacidade Vital Forçada & $3,74(\mathrm{~L}$ & $3,18(\mathrm{~L})$ & $3,17(\mathrm{~L})$ \\
\hline $\begin{array}{l}\text { Volume Expiratório Força- } \\
\text { do no Primeiro Segundo }\end{array}$ & $3,24(\mathrm{~L}$ & $2,42(\mathrm{~L})$ & $2,72(\mathrm{~L})$ \\
\hline $\begin{array}{l}\text { Volume Expiratório Força- } \\
\text { do no Primeiro Segundo / } \\
\text { Capacidade Vital Forçada }\end{array}$ & $87,32(\mathrm{~L})$ & $\begin{array}{l}75,95 \\
(\mathrm{~L})\end{array}$ & $\begin{array}{l}84,24 \\
(\mathrm{~L})\end{array}$ \\
\hline Pico de Fluxo Expiratório & $\begin{array}{l}406,56 \\
(\mathrm{~L} / \mathrm{min})\end{array}$ & $\begin{array}{l}262,2 \\
(\mathrm{~L} / \mathrm{min})\end{array}$ & $\begin{array}{l}364,3 \\
(\mathrm{~L} / \mathrm{min})\end{array}$ \\
\hline
\end{tabular}

Tabela 1. Valores espirométricos, pré e pós-tratamento. 


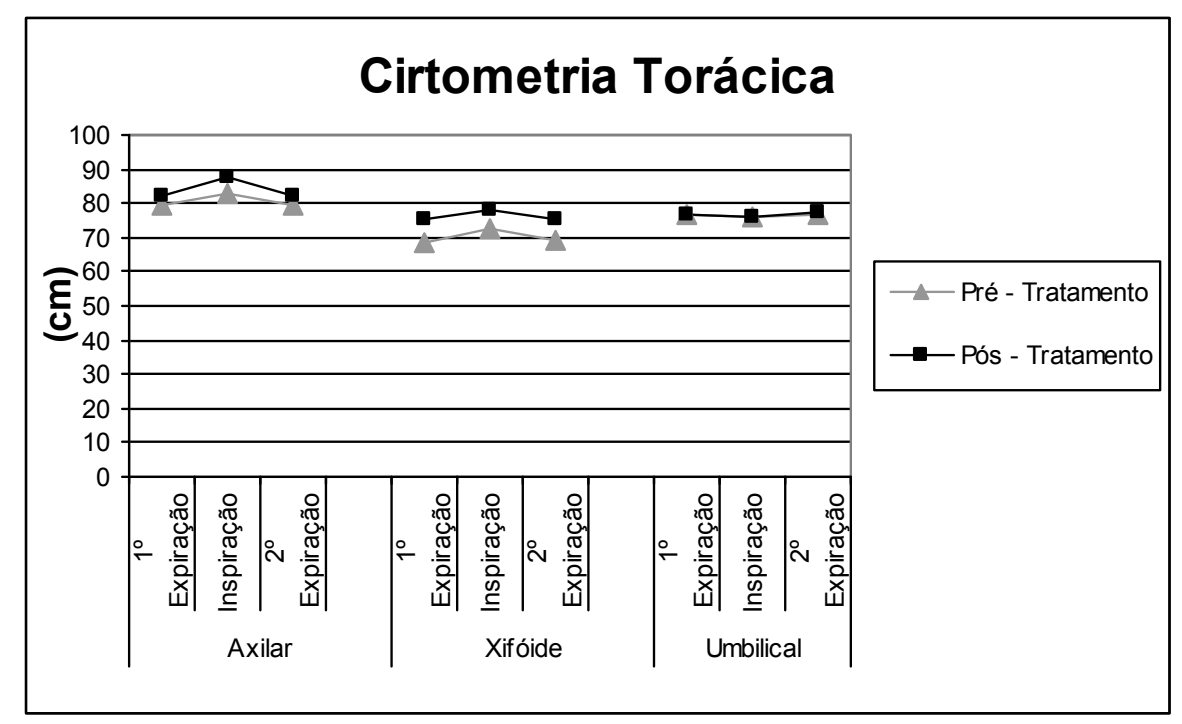

Figura 1. Valores de cirtometria torácica, pré e pós-tratament

Nos valores pré e pós-tratamento $\left(1^{\circ}\right.$ dia e $45^{\circ}$ dia, respectivamente) do AIT, observou-se um aumento de 13 pontos, e no IDB, um aumento de apenas 2 pontos. Para o QQV, no mesmo período, pode-se notar nos domínios apresentados: diminuição de $6,5 \%$ na limitação das atividades físicas devido aos sintomas da asma, diminuição de $33,4 \%$ na freqüência e gravidade dos sintomas, aumento de $16,7 \%$ na adesão ao tratamento, aumento de $8,3 \%$ no domínio sócio-econômico e diminuição de $7,1 \%$ no domínio psico-social.

As mensurações de freqüência cardíaca, freqüência respiratória e saturação, pré e póssessão, não apresentaram alterações clínicas.

\section{DISCUSSÃO}

Considerando os resultados obtidos no presente estudo, a aplicação do Método Watsu sugere ser benéfico com relação à mobilidade torácica, ventilação, estado de ansiedade e qualidade de vida do indivíduo asmático.

Analisando os valores da cirtometria torácica, ocorreu uma melhora na mobilidade do gradil costal, devido aos aumentos dos diâmetros de caixa torácica durante a inspiração e diminuição durante a expiração, nas medidas axilar e xifóide. Isto sugere um maior relaxamento muscular, uma maior mobilidade das estruturas ósseas, principalmente nas articulações costo-esternais e costo-vertebrais.

Os movimentos rotacionais de tronco realizados no método Watsu geram dissociação de cintura pélvica e escapular, alongamento de cadeia muscular cruzada, mobilidade das articulações costo-esternais e costo-vertebrais.

Associado à imersão ao nível dos ombros, a caixa torácica e o abdome sofrem uma compressão devido à pressão hidrostática, gerando alongamento dos músculos inspiratórios, diafragma e intercostais externos ${ }^{12}$. Um estudo com indivíduos asmáticos em imersão associado a exercícios respiratórios mostrou alongamento de musculatura inspiratória ${ }^{13}$. Outro estudo apresentou ganho na expansibilidade torácica após um programa de exercícios de fisioterapia aquática (alongamentos, mobilização passiva de tronco e fortalecimento) em indivíduos com espondilite anquilisante ${ }^{14}$.

A capacidade vital, durante a imersão, reduz aproximadamente 5 a $10 \%$, isto ocorre devido ao aumento do volume sangüíneo intratorácico. Além disso, a pressão hidrostática exerce uma pressão sobre o abdome, elevando o diafragma e gerando restrição para as estruturas torácicas ${ }^{15,16}$. No presente estudo, a CVF teve uma leve diminuição $(1 \mathrm{~mL})$, sem repercussão clínica.

A obstrução das vias aéreas é caracterizada por redução do VEF1 inferior a $80 \%$ do previsto1. Ao final do tratamento, foi observado um valor de VEF1 de $83 \%$ (2,72L) do previsto, o que não indica obstrução brônquica. Os valores de VEF1/CVF também não apresentaram indicativo de obstrução brônquica. Ao expirar com o corpo em imersão, pode-se aumentar a pressão do tracto respiratório e prevenir o colapso das pequenas vias aéreas ${ }^{17}$. 
O Método Watsu sugere ser benéfico para o estado de AIE, por proporcionar um estado de relaxamento profundo, apresentando benefícios para a saúde mental e física, além de aprimorar a habilidade do indivíduo em lidar com situações de estresse, tensão e ansiedade ${ }^{7,18}$. O número reduzido de terapias pode não ter sido o suficiente para alterar o resultado da AIT. O tratamento para condições de transtornos de ansiedade influencia positivamente o prognóstico da doença respiratória e melhora substancialmente a qualidade de vida desses pacientes ${ }^{19}$.
No presente estudo, a técnica sugere ser benéfica na qualidade de vida. $O$ Watsu promove uma nova perspectiva e mais aceitação diante da vida e em relação às outras pessoas ${ }^{7}$, contribuindo para que indivíduo aceite suas limitações.

O Método Watsu sugere ser benéfico neste estudo de caso, com relação à mobilidade torácica, ventilação, ansiedade e qualidade de vida. Porém, é necessário propor maior número de sessões a serem aplicadas em um número maior de indivíduos para confirmar estatisticamente os resultados positivos alcançados nesta pesquisa.

\section{REFERÊNCIAS}

1.Consenso Brasileiro no Manejo da Asma. J Pneumol 2002;24:1-46.

2.Mauad T, Souza ASL, Saldiva PHN, Dolhnikoff M. Remodelamento brônquico na asma. J Pneumol 2000;26:91-8.

3.Stirbulov R. Epidemiologia da asma. Bol Pneumol Paul 2006;19:6-8.

4.Godoy DV, Godoy RF. Redução nos níveis de ansiedade e depressão de pacientes com doença pulmonar obstrutiva crônica (DPOC) participantes de um programa de reabilitação pulmonar. J Pneumol 2002;28:120-4.

5.Guerrero VN, Mazzotti G, Villaran C, Caceres D. Prevalencia y correlaciones de trastornos de ansiedad generalizada: Depresivo mayor y de panico en pacientes asmáticos adultos según grado de severidad. Rev Neuropsiquiatr 2001;64:36-50.

6.Routi RG, Morris DM, Cole AJ. Aquatic Rehabilitation. Philadelphia: Lippincott, 1997, 463p.

7.Dull H. Watsu - exercícios para o corpo na água. São Paulo: Summus, 2001, 224p.

8. Kakizaki F, Shibuya M, Yamazaki T, Yamada M, Suzuki H, Homma I. Preliminary report on the effects of respiratory muscle stretch gymnastics on chest wall mobility in patients with chronic obstructive pulmonary disease. Respir Care 1999; 44: 409-14.

9.Fernandez ALG, Cabral AL, Faresin SM. I Consenso Brasileiro de Educação em Asma. J Pneumol 1996;22:1-24.

10.Gorenstien C, Zuardi AW. Escalas de avaliação clínica em psiquiatria e psicofarmacologia. São Paulo: Lemos, 2000, 438p. 11.Spilberger CD, Goursush RL, Lushene RE. Inventário de Ansiedade Traço - Estado - IDATE, Rio de Janeiro: CEPA - Centro Editor de Psicologia Aplicada LTDA, 1979, 60p.
12.Pereira KS, Cubero IM. Alterações fisiológicas do sistema pulmonar durante a imersão. Rev Fisioter UNICID 2000;1: 8390.

13.Pereira KS. Estudos dos efeitos da imersão em piscina aquecida no sistema cardio-respiratório de pacientes asmáticos após a aplicação de um programa de reabilitação pulmonar [Tese]. São Paulo: FMUSP, 2005, 115p.

14.Ide RM, Onaka GM, Casarolli LM, Centenaro LA, Caromano FA. Flexibilidade, mobilidade torácica e força muscular respiratória de sujeitos com espondilite anquilosante submetidos um programa de exercícios de fisioterapia aquática. Salusvita 2005;24:29-41.

15.Anstey K, Roskell C. Hydrotherapy: Detrimental or beneficial to the respiratory system? Physiother 2000;86:5-13.

16. Hall J, Bisson D, O'Hare P. The Phyisiology of Immersion. Physiother 1990;76:517-21.

17.Kurabayashi H, Machida I, Handa H, Akita T, Kubota K. Comparison of three protocols for breathing exercises during immersion in $38^{\circ} \mathrm{C}$ water for chronic obstructive pulmonary disease. Am J Phys Med Rehab 1998;77:145-8.

18.Cunha MG, Caromano FA. Efeitos fisiológicos da imersão e sua relação com a privação sensorial e o relaxamento em hidroterapia. Rev Ter Ocup 2003;14:85-102.

19.Isolan L, Nogueira L, Kipper L, Manfro GG, Barreto SM. Associação entre transtorno do pânico, asma e outras doenças obstrutivas pulmonares. Rev Psiquiatr Clin 2001;28:60-7. 